\title{
Prevention of Cardiovascular Risk Factors in the First Place
}

\author{
Darwin R. Labarthe
}

\begin{abstract}
New emphasis is placed in preventive cardiology on the concept of prevention of cardiovascular (CVD) risk factors in the first place: Success would make the risk factors themselves rare in the population, and even early pre-adult atherosclerosis would become less common and less extensive. This concept raises several practical considerations which will be illustrated by selected data. (1) Risk factor prevention includes "primordial prevention," conceived by Strasser; but where primordial prevention is too little or too late (the usual case?), prevention of the risk factors must address the already-affected population. (2) Risk factor prevention includes an important focus on youth but must extend to both earlier and later ages to achieve its goals; this is because, on the one hand, the risk factors and atherosclerosis may be advanced already during youth and, on the other hand, risk factor development progresses well beyond youth. (3) Risk factor prevention requires that several socially institutionalized gaps be bridged --such as those between childhood and adulthood, school and workplace, or pediatrics and adult medicine; continuity of preventive policies and practices is needed instead. (4) Risk factor prevention depends on better understanding of the determinants and early development of the risk factors; causal relations between preconditions for risk factor development and progression of the risk factors themselves need to be firmly established, just as the risk factors have long since become strongly linked to CVD events. (5) Risk factor prevention should be measured by reductions achieved in rates of progression of the risk factors, in either their absolute levels or rates of change with age, or in the frequency with which they reach levels requiring treatment. (6) Risk factor prevention requires further research through both observational studies and intervention trials, to be followed by long-term demonstrations to evaluate the effectiveness and costs of intervention. J Epidemiol,1996; 6 : S65-S69.
\end{abstract}

cardiovascular diseases, risk factors, prevention, cholesterol

Prevention of atherosclerotic and hypertensive cardiovascular diseases is a public health challenge throughout the world. The principal risk factors for these diseases, especially coronary heart disease and stroke, are adverse blood lipid profiles, high blood pressure, and cigarette smoking. Current policies and practices for cardiovascular disease prevention include both high-risk and population-wide components. The highrisk approach requires detection and management of these risk factors, once established in the affected individuals. The population-wide approach uses interventions intended to shift the distribution of risk favorably in those populations in which a given risk factor has become prevalent.
By contrast, new emphasis has recently been placed on the concept of prevention of the risk factors for these cardiovascular diseases "in the first place." This concept is based on the view that the development of high risk itself among individuals may be prevented, and that the incidence and prevalence of the risk factors in the population at large may be substantially reduced, if not eradicated. The purpose of this presentation is to consider several implications of this concept.

The new impetus to prevention of the risk factors in the first place is attributable to the 1994 report of a task force appointed to advise the U.S. National Heart, Lung, and Blood Institute on future research in the epidemiology and prevention of cardio-

Epidemiology Research Center, School of Public Health, The University of Texas-Houston Health Center, Texas, U.S.A. Address for correspondence : Darwin R. Labarthe, Health Science Center, School of Public Health, The University of TexasHouston, Houston, Texas, 77030 U.S.A. 
vascular diseases. That report stated, "The strategic key, and the greatest opportunity in preventing CVD [cardiovascular diseases], is to prevent the development of CVD risk in the first place"."

Was this an altogether new concept when expressed by the Task Force? The answer of course is no, because there were important antecedents. For example, the risk factors had been studied in childhood and adolescence for more than two decades, to determine the potential to modify their early development and thereby prevent high risk in adulthood. Strategies had already been proposed to enable countries throughout the world to reduce the risks of adult cardiovascular diseases, through intervention on patterns of diet, physical activity, and tobacco use so as to reduce the burden of the major cardiovascular risk factors ${ }^{2}$.

Further, and more directly, the concept of prevention of the risk factors in the first place was anticipated by Strasser in his forecast, "Reflections on Cardiovascular Diseases," published in 19783). He wrote, "Real grass-roots prevention should start by preserving entire risk-factor-free societies from the penetration of risk factor epidemics. Here lies the possibility of averting one of tomorrow's world health problems. I wish to propose the term of protoprophylaxis or primordial prevention." It seems clear that "primordial prevention," as coined by Strasser, would be intended to prevent the risk factors in the first place. However, in many populations the opportunity for primordial prevention in this specific sense may have been missed; if such efforts were too little, or too late, would this foreclose the possibility for prevention of the risk factors in the first place?

This is the first consideration of six to be addressed: (1) the relation between prevention of the risk factors in the first place and primordial prevention; (2) the need for a broader focus than on "youth" alone, to extend prevention of the risk factors to both earlier and later periods of life ; (3) the importance of bridging gaps across age groups and periods of life to assure continuity in policy, practice and research on risk factor prevention; (4) the need for further evidence to strengthen the basis for risk factor prevention; (5) some measures by which effectiveness of strategies for risk factor prevention can be evaluated; and (6) the kinds of research needed to establish prevention of the risk factors in the first place as a major component of cardiovascular disease prevention. Each of these considerations will be addressed briefly below.

\section{(1) The Relation Between Prevention of the Risk Factors in the First Place and Primordial Prevention}

First, to put prevention of the risk factors in the first place in context, the high-risk and population-wide strategies may be contrasted: The high-risk strategy targets individuals with one or more established risk factors and aims to convert extremes of risk toward the population mean. The population-wide strategy targets whole populations irrespective of individual levels of risk and aims to shift the whole distribution of risk toward the optimum, including a shift at the mean of the distribution and not only at the extreme ${ }^{4}$. Next, the terms secondary and primary prevention have been applied both to cardiovascular events and to the risk factors, with some resulting confusion. Secondary prevention of coronary heart disease, for example, is the prevention of clinical recurrence or progression, and relief of disability, for existing coronary heart disease; secondary prevention of a risk factor, such as high total cholesterol concentration, is the treatment of that condition, by whatever means, to reduce or if possible reverse its adverse effects. Primary prevention of coronary heart disease is the prevention of clinical events by intervention against established risk factors; for a risk factor such as high total cholesterol concentration, primary prevention is prevention of the development of the factor beyond the optimal range, that is, prevention of the risk factor in the first place. Adding primordial prevention to this scheme, with Strasser's intended meaning, we have the strategy of "preserving whole risk-factor-free societies from the penetration of risk factor epidemics ${ }^{3)}$ ".

These aspects of prevention as they apply to the risk factors are represented in the Figure, by level (primordial, primary and

\section{Societal conditions \\ Risk-factor-free $\quad$ Risk factors present \\ (Whole population) Whole population High-risk individuals}

$\begin{array}{llll}\text { Primordial prevention }\left(0^{\circ}\right) & \mathrm{X} & - & - \\ \text { Primary prevention }\left(1^{\circ}\right) & & & \\ \quad \text { Population-wide } & - & \mathrm{X} & \mathrm{X}(?) \\ \quad \text { High-risk } & - & - & \mathrm{X}(?) \\ \text { Secondary prevention }\left(2^{\circ}\right) & & & \\ \quad \text { Population-wide } & - & \mathrm{X} & \mathrm{X} \\ \text { High-risk } & - & - & \mathrm{X}\end{array}$

Figure 1. Strategies for prevention of CVD risk factors. 
secondary), by applicable target (high-risk or population-wide) and by societal conditions, with respect to the persisting absence or the presence of the risk factor. Prevention of the risk factors in the first place can apply in each circumstance noted by ' $\mathrm{X}$ ' under either primordial or primary prevention. (Whether primary prevention of the risk factors has a high-risk strategy depends on having knowledge of the individual precursors of risk factor development, knowledge which requires further research in some important respects.) Secondary prevention of the risk factors has both population-wide and highrisk components, those at high risk being arbitrarily defined as having attained undesirable levels of one or another of the risk factors. Thus primordial prevention can be considered to constitute an important aspect, but only one aspect, of the range of opportunities for prevention of the risk factors in the first place.

For example, first, in some societies total cholesterol concentrations typically remain low throughout life, as was true of Japan until the most recent decades. If dietary habits which favor low cholesterol concentrations could be preserved, as is considered possible in parts of India, primordial prevention in this respect could succeed. In Japan, however, the point has now been reached where dietary patterns leading to increased cholesterol concentrations require intervention at both the population and individual levels. Undesirable levels of cholesterol concentration may be prevented, but no longer in the context of primordial prevention. Second, some populations remain in which blood pressure rises very little with age throughout adulthood; preservation of the determinants of adult blood pressure levels in these populations would constitute primordial prevention of high blood pressure, while in most populations intervention is required to counter the effects of these determinants so as to reduce the incidence of high blood pressure. Third, in the area of smoking, where existing social prohibitions of tobacco use can be maintained, despite pressures from the tobacco industry, primordial prevention of this risk factor could be achieved. But, commonly, marketing of commercial tobacco products has already become established, so that preventive efforts must seek to prevent or reverse the early steps toward nicotine addiction which are reflected in regular cigarette smoking. As in other aspects of cardiovascular disease prevention, prevention of the risk factors in the first place must be based on the existing conditions and the appropriate choice among available strategies applied.

(2) The Need for a Broader Focus than on "Youth" Alone, to Extend Risk Factor Prevention to Both Earlier and Later Periods of Life

A common view of prevention of the risk factors is that it concerns children, or adolescents, or 'youth,' exclusively. The emphasis on prevention of the risk factors in the first place suggests the need to reconsider this view. For example, the PDAY Study (Pathobiological Determinants of
Atherosclerosis in Youth) demonstrates involvement of up to 20 percent of the surface of the aorta, and to a lesser extent of the coronary arteries, with raised atheromata in decedent subjects as young as 15-19 years ${ }^{5}$. The further evidence from this and other studies that risk factor levels at this age are strongly correlated with the extent of pathology suggests that modification of risk factors well before this age is necessary to prevent significant early atherosclerosis. Whether intervention in fetal or neonatal life is necessary to afford full protection, as implied by the hypothesis of 'programming' of risk in utero or in the first year of life, remains to be established ${ }^{6}$.

It is also clear that the progressive change in risk factors to reach levels requiring individual intervention need not end with youth but extends well into adulthood. Serum cholesterol concentrations among young adults in the longitudinal study, CARDIA, in the U.S. illustrate this point ${ }^{\emptyset}$. Black and white males and females, with or without completion of high-school education, all exhibited increases in plasma total cholesterol concentration from ages 18-24 to ages $25-30$ years. From these and other examples, one may conclude that youth neither opens nor closes the period for risk factor prevention in the first place, since atherosclerosis may be advanced already within this period and risk factor development progresses well beyond it.

\section{(3) The Importance of Bridging of Gaps Across Age Groups and Periods of Life to Assure Continuity in Policy, Practice and Research on Risk Factor Prevention}

The range in ages or periods of life during which the risk factors can potentially be prevented is broad and argues for overcoming a segmented -.. or, worse, fragmented --approach to risk factor prevention. The reality of progressive risk factor development from at least early childhood into early or middle adulthood suggests the need for a revised perspective. Instead of the current framework of institutionalized gaps between childhood and adulthood, school and workplace, or pediatrics and adult medicine, continuity of research and practice in risk factor prevention across periods of life is needed.

\section{(4) The Need for Further Evidence to Strengthen the Basis for Risk Factor Prevention}

Primary prevention of coronary heart disease is based upon the well established causal relations between the major risk factors and both population rates and individual risks of coronary events. There are important linkages between these risk factors in childhood and adolescence and in adulthood: familial aggregation, or resemblance of risk factor levels within family pedigrees; familial concordance between a history of coronary events in a parent and risk factor levels in the offspring; tracking correlations between childhood or adolescence and early adulthood; and the already noted correlation between the risk factors and the pathology of atherosclerosis in the late 
teen-age years. But what are the causes of the risk factors themselves? Linkages should be even more clearly established between preconditions for risk factor development and progression of the risk factors themselves, just as the risk factors have long since become strongly linked to measures of disease occurrence.

While much is known, there are important gaps in our knowledge of the early development of the risk factors. For example, current policy for intervention on total cholesterol concentration in the U.S. assumes a constant value for screening at all ages, for both males and females, from ages 2-19 years. At age 20, the screening criterion to distinguish desirable from borderline or higher levels shifts from 170 to $200 \mathrm{mg} / \mathrm{dl}^{8)}$. Because previous epidemiologic studies, both cross-sectional and longitudinal, had indicated stronger associations of blood lipids and blood pressure with indices of growth and maturation than with age, we undertook a mixed longitudinal study of boys and girls aged 8,11 , and 14 at entry, in order to investigate development of the risk factors as growth processes ${ }^{2}$. The appropriate design included three age groups, four-monthly examinations, and overlap in age at observation between successive cohorts within the study population. A total of 678 children were enrolled, and within 4 years more than 5000 complete examinations were conducted. Both total cholesterol concentration and percent body fat have been modeled in initial longitudinal analyses.

Actually, confirming the suggestion from previous crosssectional studies, we have demonstrated that there is a dynamic change in cholesterol concentrations in this period of life which strongly influences the expected yield of screening by both age and gender. Further, there is corresponding change in percent body fat, only for boys; for girls there is no apparent relation between total cholesterol concentration and percent body fat. Therefore, the relation between fatness and blood lipids cannot be the same for both boys and girls, and intervention strategies aimed at modifying blood lipids through control of obesity cannot be expected to have the same effects in both groups. Clearly, further understanding of the natural history of the development of blood lipids in adolescence is needed to refine recommendations for intervention to prevent this risk factor in the first place.

\section{(5) Some Measures by which Effectiveness of Strategies for Risk Factor Prevention can be Evaluated}

How are we to judge the effectiveness of interventions for prevention of the risk factors in the first place? When the progression of the risk factors to levels requiring treatment is taken as the endpoint, either the incidence of this event or the rate of progression from earlier levels of the risk factor is an appropriate response variable for evaluating the effectiveness of intervention. The public health burden of detection and treatment of established risk factors, coupled with the causal relation of the risk factors with adult cardiovascular diseases, makes the choice of risk factor change as an endpoint appropriate. Studies with early intervention and longer than previous follow-up, into early or middle adult ages, will provide the most rigorous assessment of the effectiveness of such interventions. An initial phase of shorter-term evaluation with successive cohorts as in the design for Project HeartBeat! can help to determine the optimum ages at intervention for one or another risk factor, perhaps differing between males and females or by other group characteristics.

In general, however, the efficacy and effectiveness of preventive strategies should be measured by the reductions they achieve in the rates of progression of the risk factors, by which they either reach treatable levels or enter lesser, but also undesirable, categories. For example, the progression of levels of blood cholesterol concentration continues from the 20 s to the $50 \mathrm{~s}$. The prevalence of desirable levels of cholesterol concentration is still 65-69 percent in U.S. men and women aged from 20-34 years, and declines to $30-36$ percent only by age $45-54$ years ${ }^{10)}$. Maintenance of higher proportions of the population in the desirable range at successively older ages would constitute strong evidence of the effectiveness of interventions to prevent the risk factors in the first place.

\section{(6) The Kinds of Research Needed to Establish Prevention of the Risk Factors in the First Place as a Major Component of Cardiovascular Disease Prevention}

It was suggested by Nobel laureates Michael Brown and Joseph Goldstein, in an editorial in Science in May 1996 that coronary heart disease was about to disappear as a major public health problem ${ }^{11)}$.The fallacy of this viewpoint was noted in subsequent correspondence to Science ${ }^{12)}$ :The evidence to the contrary includes the demographic forces which maintain the predominance of coronary heart disease as a cause of death in the U.S., even though age-specific rates have decreased by more than 50 percent since the peak of the U.S. coronary epidemic in the 1960s. It also includes the cultural factors underlying the upsurgence of coronary heart disease in the more affluent socioeconomic strata in developing countries. Finally, it includes the necessarily limited role of lipid-lowering drugs in prevention of coronary heart disease, since they cannot benefit those who have already died at the first manifestation of coronary heart disease, and even in the most favorable conditions of clinical trials they failed to prevent the majority of new or recurring coronary events. These considerations not only demonstrate the reality of coronary heart disease as a public health problem for the foreseeable future; they also add to the rationale of the Task Force on Research in Epidemiology and Prevention of Cardiovascular Diseases in identifying the prevention of the risk factors in the first place as the greatest opportunity in preventing cardiovascular diseases ${ }^{1)}$.

Much remains to be contributed by epidemiology to the sci- 
entific basis for prevention of the risk factors in the first place. This includes further detailed observational studies of the course of development of the risk factors, across the period from conception to middle adulthood, in diverse populations. It also includes small clinical experiments and larger clinical trials to determine the optimum methods and timing of interventions. Finally, it includes field demonstrations and evaluations of preventive strategies, for their effectiveness as measured against the expected rates of progression of the risk factors to treatable levels.

The several considerations discussed here suggest the importance of a change in viewpoint toward prevention of the risk factors in the first place, in contrast to reliance entirely on their secondary prevention through the currently proposed high-risk and population-wide approaches to existing risk factors. The suggested research agenda needs strong support by the agencies responsible for the public's health. This support will be forthcoming only to the extent that the epidemiologic community advocates this need, in the interest of prevention of cardiovascular diseases as a continuing worldwide challenge to public health.

\section{REFERENCES}

1. National Heart, Lung, and Blood Institute. Report of the Task Force on Research in Epidemiology and Prevention of Cardiovascular Diseases. US Department of Health and Human Services, Public Health Service, National Institutes of Health, August 1994.

2. WHO Expert Committee. Prevention in Childhood and Youth of Adult Cardiovascular Diseases: Time for Action. Technical Report Series 792. World Health Organization, Geneva, 1990.

3. Strasser T. Reflections on cardiovascular diseases. Interdisc Sci Rev 1978; 3:225-230.

4. Rose G., Sick individuals and sick populations. Int J Epid 1985; 14:32-38.

5. Pathobiological Determinants of Atherosclerosis in Youth
(PDAY) Research Group. Relationship of atherosclerosis in young men to serum lipoprotein cholesterol concentrations and smoking. A preliminary report from the Pathobiological Determinants of Atherosclerosis in Youth (PDAY) Research Group. JAMA 1990; 264:3018-3024.

6. Barker DJP, ed. Fetal and Infant Origins of Adult Disease. British Medical Journal, London, 1992.

7. Cutter GR, Burke GL, Dyer AR, Friedman GD, Hilner JE, Hughes GH, Hulley SB, Jacobs Jr DR, Liu K, Manolio TA, Oberman A, Perkins LL, Savage PJ, Serwitz JR, Sidney S, Wagenknecht LE. Cardiovascular Risk Factors in Young Adults. The CARDIA Baseline Monograph. Cont Clin Trials 1991; 12(Suppl 1):1S-77S.

8. National Cholesterol Education Program Expert Panel on Blood Cholesterol Levels in Children and Adolescents. Report of the Expert Panel on Blood Cholesterol Levels in Children and Adolescents. US Department of Health and Human Services, Public Health Service, National Institutes of Health, National Heart, Lung, and Blood Institute, National Cholesterol Education Program. NIH Publication 1991; No. 91-2732.

9. Labarthe DR, Harrist RB, Dai S, Grunbaum JA, Nichaman MZ. Study of changes in cardiovascular risk factors in adolescence as growth processes: Dynamics of total cholesterol concentration in Project HeartBeat! (Abstract). Am J Epid 1996; 143(Suppl): S49.

10. Expert Panel on Detection, Evaluation, and Treatment of High Blood Cholesterol in Adults. Summary of the Second Report of the National Cholesterol Education Program (NCEP) Expert Panel on Detection, Evaluation, and Treatment of High Blood Cholesterol in Adults (Adult Treatment Panel II). JAMA 1993; 269:30153023.

11. Brown MS, Goldstein JL. Heart attacks: Gone with the century? (editorial) Science 1996; 272:629.

12. Labarthe DR. Battling heart disease. (letter) Science 1996; 273. 